\title{
Mesoporous SBA-15 nanoparticles: An efficient and eco-friendly Catalyst for one- pot synthesis of 3, 4-dihydropyrimidin-2(1H)-ones under solvent-free conditions
}

\author{
Yaghoub Sarrafi ${ }^{*}$, Farzane Pazokie, Seyed Naser Azizi, Kamal Alimohammadi and Ebrahim Mehrasbi, \\ Elham Chiani
}

Department of Organic Chemistry, Faculty of Chemistry, University of Mazandaran, Babolsar, Iran

\section{H R O N I C L E}

Article history:

Received June 28, 2013

Received in Revised form

December 10, 2013

Accepted 20 December 2013

Available online

21 December 2013

Keywords:

Biginelli condensation

mesoporous

3, 4-dihydropyrimidin-2(1H)-ones

Heterogeneous catalysis

\author{
A B S T R A C T \\ A simple and efficient procedure for the synthesis of 3, 4-dihydropyrimidin-2(1H)-ones under \\ solvent-free condition using non-toxic and mild acid mesoporous SBA-15 nanoparticles as a \\ catalyst has been investigated. This method has the advantages of the excellent yield, short \\ reaction time, environmentally friendly conditions and simple experimental procedure.
}

\section{Introduction}

In recent decades there was an exponential growth in the application of inorganic solid acid catalysts in order to carry out organic synthesis because of its importance in terms hazardous chemicals in the present industrial scenario ${ }^{1}$. Inorganic solid catalysts such as SBA-15 are interesting for organic synthesis since they are environmental protection ${ }^{2}$. Mesoporous SBA-15 nanoparticles have a highly ordered hexagonal structure, large pore, high surface area, high thermal stability and thicker walls in comparison with Mobile composition of matter number 41 (MCM-41) ${ }^{3}$. Green chemistry approaches hold out significant potential not only for save energy, prevent solvent wastes, hazards and toxicity but also in development of new methodologies towards previous unobtainable materials, using existing technologies ${ }^{4}$. The elimination of volatile organic solvents in green chemistry is desirable in order to reduce the amount of residual solvent and atmospheric pollution. However using solvent-free organic reactions can reduce waste products and energy costs of synthesis ${ }^{5}$.

* Corresponding author. Tel/Fax:+98-1125342350

E-mail addresses: ysarrafi@umz.ac.ir (Y. Sarrafi) 
Biginelli reaction, discovered in 1893 , for the synthesis of multi-functionalized 3, 4dihydropyrimidin-2(1H)-one (DHPMs) by 1, 3-dicarbonyl compounds, benzaldehyde, and urea in ethanol solution with a catalytic amount of $\mathrm{HCl}$ at reflux ${ }^{6}$. DHPMs and their derivatives have attracted considerable interest because of their therapeutic and pharmacological properties such as antiviral, antibacterial, antihypertensive and antitumor effects ${ }^{7-10}$. In recently years, in order to prepare DHPMs, due to importance of this compounds, several synthetic procedures have been investigated including classical conditions, using Lewis acids as well as protic acid promoters such as concd $\mathrm{HCl}^{11}$, concd $\mathrm{H}_{2} \mathrm{SO}_{4}{ }^{12}$, p-sulfonic acid calixarenes ${ }^{13}$, zeolites like HZSM-5, Hy, MCM-41 ${ }^{14}$, zeolites Na-beta ${ }^{15}$, natural HEU type zeolite $^{2}$ and functionalized SBA-15 in different organic solvents $^{16,17}$.

However, some of these methods have drawbacks such as the need of strong and protic acids ${ }^{11,12}$, toxic solvent ${ }^{15}$, longer reaction time, tedious workup, environmental disposal problems ${ }^{13,16-17}$ and lower yields of the products ${ }^{2}$. Therefore, the utilization of a simple and environmentally friendly method for the preparation of 3, 4-dihydropyrimidin-2-(1H)-ones is of prime importance. Cause of emerging importance of mesoporous SBA-15 nanoparticles as a catalyst that is related to our research for reactions proceeding in solvent-free condition ${ }^{18,19}$, we would report a simple and facile synthesis of dihydropyrimidiones by using mesoporous SBA-15 nanoparticles as a heterogeneous and ecofriendly catalyst under solvent-free conditions in high yields.

\section{Experimental}

\subsection{Catalyst preparation}

Amorphous Silica was extracted from Stem Sweep Ash (SSA) by a suitable alkali solution with approximately $80 \%$ purity, converted to silicate solution with $\mathrm{SiO}_{2} / \mathrm{Na}_{2} \mathrm{O}=2.88$ and used as silica source for the synthesis of mesoporous SBA-15 nanoparticles by a sol-gel method in the presence of non-ionic block co-polymer (P123) as co-template ${ }^{20}$. Mesoporous SBA-15 nanoparticles was acidified by ammonium chloride $(1 \mathrm{M})$ then calcined at $550{ }^{\circ} \mathrm{C}$ for $5 \mathrm{~h}$.

2.2 Synthesis of dihydropyrimidiones derivatives

A mixture of aldehyde (1 mmol), 1, 3-dicarbonyl $(1.5 \mathrm{mmol})$, urea or thiourea $(1.5 \mathrm{mmol})$ and mesoporous SBA-15 nanoparticles $(0.1 \mathrm{gr})$ was heated at $100{ }^{\circ} \mathrm{C}$ under stirring. The reaction was monitored by TLC using ethyl acetate: hexane (4:6) as eluent. After completion, the reaction mixture was cooled, poured into cold water and stirred for $5 \mathrm{~min}$. The solid was suction filtered and the filtrate was evaporated and the residual solid was washed with cold water $(20 \mathrm{~mL} \times 2 \mathrm{~mL})$ then recrystallized in ethyl acetate or ethanol to afford pure product.

Dimethyl 2-(4-(5-(ethoxycarbonyl)-6-methyl-2-oxo-1,2,3,4-tetrahydropyrimidin-4yl)phenoxy)maleate (Entry 14): ${ }^{1} \mathrm{H}$ NMR $\left(400 \mathrm{MHz}, \mathrm{DMSO}-d_{6}\right): \delta=1.10\left(\mathrm{t}, J=6.8 \mathrm{~Hz}, 3 \mathrm{H}, \mathrm{CH}_{3}\right), 2.24$ $\left(\mathrm{s}, 3 \mathrm{H}, \mathrm{CH}_{3}\right), 3.61\left(\mathrm{~s}, 3 \mathrm{H}, \mathrm{CH}_{3}\right), 3.70\left(\mathrm{~s}, 3 \mathrm{H}, \mathrm{CH}_{3}\right), 4.01\left(\mathrm{~m}, 2 \mathrm{H}, \mathrm{CH}_{2}\right) 5.15(\mathrm{~d}, \mathrm{~J}=3.2 \mathrm{~Hz}, 1 \mathrm{H}, \mathrm{CH})$, $6.63(\mathrm{~s}, 1 \mathrm{H}, \mathrm{CH}), 6.90\left(\mathrm{~d}, \mathrm{~J}=8.6 \mathrm{~Hz}, 2 \mathrm{H}, \mathrm{CH}_{\mathrm{Ar}}\right), 7.18\left(\mathrm{~d}, \mathrm{~J}=8.6,2 \mathrm{H}, \mathrm{CH}_{\mathrm{Ar}}\right), 7.73($ br s, $1 \mathrm{H}, \mathrm{NH})$, 9.20 (br s, $1 \mathrm{H}, \mathrm{NH}) .{ }^{13} \mathrm{CNMR}\left(100 \mathrm{MHz}, \mathrm{DMSO}-d_{6}\right): \delta=14.5,18.2,52.3,53.6,53.8,59.7,99.7,116.0$, $128.2,140.2$, 148.8, 148.9, 152.5, 155.8, 162.3, 163.6, 165.8. IR (KBr): v=3310, 1720, 1678, 1657 , $1439,1376 \mathrm{~cm}^{-1}$.

Dimethyl 2- (2- (5- acetyl- 6- methyl- 2- oxo- 1, 2, 3, 4- tetrahydropyrimidin- 4- yl) phenoxy) maleate (Entry 16): ${ }^{1} \mathrm{H}$ NMR $\left(400 \mathrm{MHz}, \mathrm{DMSO}-d_{6}\right): \delta=2.06\left(\mathrm{~s}, 3 \mathrm{H}, \mathrm{CH}_{3}\right), 2.32\left(\mathrm{~s}, 3 \mathrm{H}, \mathrm{CH}_{3}\right), 3.66$ $\left(\mathrm{s}, 3 \mathrm{H}, \mathrm{CH}_{3}\right), 3.71\left(\mathrm{~s}, 3 \mathrm{H}, \mathrm{CH}_{3}\right), 5.68(\mathrm{~d}, J=3.2,1 \mathrm{H}, \mathrm{CH}), 6.68(\mathrm{~s}, 1 \mathrm{H}, \mathrm{CH}), 6.76\left(\mathrm{~m}, 1 \mathrm{H}, \mathrm{CH}_{\mathrm{Ar}}\right), 6.85$ (br s, $1 \mathrm{H}, \mathrm{NH}) 7.06\left(\mathrm{~m}, 1 \mathrm{H}, \mathrm{CH}_{\mathrm{Ar}}\right), 7.13\left(\mathrm{~m}, 1 \mathrm{H}, \mathrm{CH}_{\mathrm{Ar}}\right) 7.24\left(\mathrm{~m}, 1 \mathrm{H}, \mathrm{CH}_{\mathrm{Ar}}\right) 9.27$ (br s, $\left.1 \mathrm{H}, \mathrm{NH}\right)$ ${ }^{13} \mathrm{CNMR}\left(100 \mathrm{MHz}, \mathrm{DMSO}-d_{6}\right): \delta=19.2,30.1,50.0,52.5,53.7,106.9,114.5,116.3,123.8,127.9$, 
$129.6,131.2,148.9,149.5,152.4,154.0,162.4,163.8,194.7$. IR $(\mathrm{KBr}): \mathrm{v}=3348,1715,1699,1613$, $1340,1325 \mathrm{~cm}^{-1}$.

\section{Results and Discussion}

At first, mesoporous SBA-15 nanoparticles with rod like pore structure was synthesized from rice husk $(\mathrm{RiH})$ using the SSA as silicon source and sol-gel method. We began to extract. The crystal like external morphology of the particle, determined by SEM. crystallite size observed from this technique is $82 \mathrm{~nm}$. The SEM image reveals agglomeration of rod-like shape SBA-15 particles ${ }^{20}$.

The approach involved the use of synthesized mesoporous SBA-15 nanoparticles from RHA for synthesis of dihydropyrimidiones under solvent-free conditions. Therefore, this method provides an efficient route for synthesis of dihydropyrimidiones which saves energy, and prevents from solvent wasting, hazards, and toxicity. The reaction conditions were initially optimized by considering three component condensations of a variety of aromatic aldehydes carrying electron donating or electronwithdrawing substituents, ethyl acetoacetate and urea to afford the corresponding product as a model reaction under various reaction conditions. The best results were obtained using 0.04gr of mesoporous SBA-15 nanoparticles, $1.5 \mathrm{mmol}$ of urea, ethylaceto acetate and $1 \mathrm{mmol}$ of aldehyde, at $100^{\circ} \mathrm{C}$ and under solvent free conditions Scheme 1. To illustrate the efficiency of the catalyst in this reaction, when the same reagents and conditions were used to carry out the reaction without catalyst, the reaction failed to give the desired product, even after a long reaction time (Table 1, entry 1).

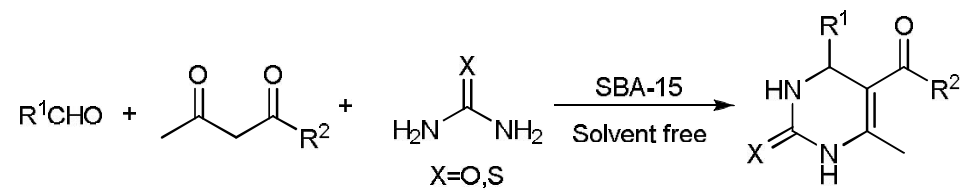

Scheme 1. Synthesis of 3, 4- dihydropyrimidin- 2 (1H)- ones using mesoporous SBA-15 nanoparticles

Table 1. Synthesis of 3, 4-dihydropyrimidin- 2(1H)- ones using mesoporous SBA-15 nanoparticles under solvent- free conditions at $100^{\circ} \mathrm{C}$

\begin{tabular}{|c|c|c|c|c|c|c|c|}
\hline \multirow[b]{2}{*}{ Entry } & \multirow[b]{2}{*}{$\mathrm{R}^{1}$} & \multirow[b]{2}{*}{$\mathrm{R}^{2}$} & \multirow[b]{2}{*}{$\mathrm{X}$} & \multirow[b]{2}{*}{$\mathrm{T}(\mathrm{min})$} & \multirow[b]{2}{*}{ Yield (\%) } & \multicolumn{2}{|c|}{$\mathrm{mp}\left({ }^{\circ} \mathrm{C}\right)$} \\
\hline & & & & & & Found & ref \\
\hline 1 & $4-\mathrm{CH}_{3}-\mathrm{C}_{6} \mathrm{H}_{4}$ & OEt & $\mathrm{O}$ & 20 & 88 & $205-206$ & $205-206^{21}$ \\
\hline 2 & $\mathrm{C}_{6} \mathrm{H}_{5}$ & OEt & $\mathrm{O}$ & 20 & 94 & $210-211$ & $209-210^{22}$ \\
\hline 3 & $3-\mathrm{Br}-\mathrm{C}_{6} \mathrm{H}_{4}$ & $\mathrm{OMe}$ & S & 50 & 65 & $185-188$ & $185-187^{23}$ \\
\hline 4 & $2,4-\mathrm{CH}_{3}-\mathrm{C}_{6} \mathrm{H}_{4}$ & $\mathrm{OMe}$ & $\mathrm{O}$ & 38 & 70 & $245-246$ & $245-247^{24}$ \\
\hline 5 & $\mathrm{C}_{6} \mathrm{H}_{5}$ & OEt & $\mathrm{S}$ & 42 & 92 & 204-205 & $205-206^{25}$ \\
\hline 6 & $4-\mathrm{OCH}_{3}-\mathrm{C}_{6} \mathrm{H}_{4}$ & OEt & $\mathrm{O}$ & 65 & 88 & $209-210$ & $205-207^{26}$ \\
\hline 7 & $4-\mathrm{OCH}_{3}-\mathrm{C}_{6} \mathrm{H}_{4}$ & $\mathrm{Me}$ & $\mathrm{O}$ & 60 & 90 & $178-179$ & $178-180^{7}$ \\
\hline 8 & $\mathrm{C}_{6} \mathrm{H}_{5}$ & $\mathrm{Me}$ & $\mathrm{O}$ & 20 & 83 & $230-231$ & $229-231^{26}$ \\
\hline 9 & $4-\mathrm{OCH}_{3}-\mathrm{C}_{6} \mathrm{H}_{4}$ & OEt & $\mathrm{S}$ & 65 & 90 & $140-141$ & $140^{27}$ \\
\hline 10 & $4-\mathrm{Cl}-\mathrm{C}_{6} \mathrm{H}_{4}$ & OEt & $\mathrm{O}$ & 60 & 87 & $218-219$ & $216-217^{22}$ \\
\hline 11 & $4-\mathrm{F}-\mathrm{C}_{6} \mathrm{H}_{4}$ & OEt & $\mathrm{O}$ & 60 & 89 & $180-181$ & $182^{26}$ \\
\hline 12 & $3-\mathrm{OCH}_{3}-\mathrm{C}_{6} \mathrm{H}_{4}$ & OEt & $\mathrm{O}$ & 50 & 91 & $210-211$ & $208^{28}$ \\
\hline 13 & $4-\mathrm{Br}-\mathrm{C}_{6} \mathrm{H}_{4}$ & $\mathrm{Me}$ & $\mathrm{O}$ & 22 & 82 & $232-233$ & $232-233^{29}$ \\
\hline 14 & $4-\mathrm{C}_{2} \mathrm{H}\left(\mathrm{CO}_{2} \mathrm{CH}_{3}\right)_{2}-\mathrm{C}_{6} \mathrm{H}_{4}$ & OEt & $\mathrm{O}$ & 60 & 88 & $222-223$ & - \\
\hline 15 & $3-\mathrm{Cl}-\mathrm{C}_{6} \mathrm{H}_{4}$ & OEt & $\mathrm{O}$ & 20 & 67 & $195-196$ & $193-195^{30}$ \\
\hline 16 & $2-\mathrm{C}_{2} \mathrm{H}\left(\mathrm{CO}_{2} \mathrm{CH}_{3}\right)_{2}-\mathrm{C}_{6} \mathrm{H}_{4}$ & $\mathrm{Me}$ & $\mathrm{O}$ & 75 & 65 & $163-165$ & - \\
\hline
\end{tabular}




\section{Conclusions}

In summary, we have developed a simple, rapid and efficient procedure for the synthesis of 3, 4dihydropyrimidine-2 $(1 H)$-one derivatives by using mesoporous SBA-15 nanoparticles as catalyst under solvent free condition. This method offers several advantages such as green reaction conditions, short reaction time, high yields and simple work-up procedure use of environmental friendly catalyst.

\section{Acknowledgements}

Financial support of this work from the Research Council of Mazandaran University is gratefully acknowledged.

\section{References}

1. (a) Corma, A. (1997). From microporous to mesoporous molecular sieve materials and their use in catalysis. Chem. Rev., 97, 2373-2420; (b) Clark, J. H., Macquarrie, D. J. (1996). Environmentally friendly catalytic methods. Chem. Soc. Rev., 25, 303-310.

2. Tajbakhsh, M., Mohajerani, B., Heravi, M. M., et al. (2005). Natural HEU type zeolite catalyzed Biginelli reaction for the synthesis of 3, 4- dihydropyrimidin-2 $(1 H)$ one derivatives. J. Mol. Catal. A: Chem., 236, 216-219.

3. Hoang, V. D., Dang, T. P., Dinh, Q. K., et al. (2010). The synthesis of novel hybrid thiolfunctionalized nano-structured SBA-15. Adv. Nat. Sci.: Nanosci. Nanotechnol., 1, 1-6.

4. Evans, D. A., Tedrow, J. S., Shaw, J. T., et al. (2002). Diastereoselective Magnesium HalideCatalyzed anti-Aldol Reactions of Chiral N-Acyloxazolidinones. J. Am. Chem. Soc., 124, 392-393.

5. Tanaka, K. (2009). Solvent-free Organic Synthesis. WILEY-VCH Verlag GmbH \& Co. KGaA, Weinheim,

6. Ramon, D. J., Yus, M. (2005). Asymmetric Multicomponent Reactions (AMCRs): The New Frontier. Angew. Chem., Int. Ed., 44, 1602-1634.

7. Ma, Y., Qian, C., Wang, L., et al. (2000). Lanthanide Triflate Catalyzed Biginelli Reaction. OnePot Synthesis of Dihydropyrimidinones under Solvent- Free Conditions. J. Org. Chem., 65, 38643868 .

8. Hu, E. H., Sidler, D. R., Dolling, U. H. (1998). Unprecedented Catalytic Three Component OnePot Condensation Reaction: An Efficient Synthesis of 5-Alkoxycarbonyl- 4-aryl-3, 4dihydropyrimidin-2(1H)-ones. J. Org. Chem., 63, 3454-3457.

9.Snider, B. B., Shi, Z. (1993). Biomimetic synthesis of ( \pm )-crambines A, B, $C_{1}$, and $C_{2}$. Revision of the structure of crambines B and $\mathrm{C}_{1}$. J. Org. Chem., 58, 3828-3839.

10. Fabian, W. M. F., Semones, M. A. (1997). Conformational analysis of 4-aryldihydropyrimidine calcium channel modulators. A comparison of ab initio, semiempirical and Xray crystallographic studies. Tetrahedron, 53, 2803-2816.

11. Biginelli, P. (1893). Synthesis of dihydropyrimidinones. Gazz. Chim. Ital., 23, 360-413.

12. Hassani, Z., Islami, M. R., Kalantari, M. (2006). An efficient one-pot synthesis of octahydroquinazolinone derivatives using catalytic amount of $\mathrm{H}_{2} \mathrm{SO}_{4}$ in water. Bioorg. Med. Chem. Lett., 16, 4479-4482.

13. Silva, D. L. da., Fernandes, S. A., Sabino, A. A., et al. (2011). P-sulfonic acid calixarenes as efficient and reusable organocatalysts for the synthesis of 3, 4-dihydropyrimidin-2(1H)-ones/thiones. Tetrahedron Lett., 52, 6328-6330. 
14.Radha, Rani .V., Srinivas, N., Radha, Kishan. M., et al. (2001). Zeolite-catalyzed cyclocondensation reaction for the selective synthesis of 3, 4-dihydropyrimidin-2(1H)-ones. Green Chem., 3, 305-306.

15. Mistry, S. R., Joshi, R. S., Sahoo, S. K. (2011). Synthesis of dihydropyrimidiones using large pore zeolites. Catal. Lett., 141, 1541-1547.

16. a) $\mathrm{Xu}, \mathrm{L}-\mathrm{W}$., Yang, M-S., Jiang, J-X., et al. (2007). Ionic liquid-functionalized SBA-15 mesoporous material: efficient heterogeneous catalyst in versatile organic reactions. Cent. Eur. J. Chem., 5, 1073- 1083; b) mondal, J., Sen, T., Bhaumik, A. (2012). Fe3O4@mesoporous SBA-15: a robust and magnetically recoverable catalyst for one-pot synthesis of 3,4-dihydropyrimidin2(1H)-ones viathe Biginelli reaction. Dalton trans., 41, 6173-6181.

17. Srivastava, R. (2010). Assessment of the catalytic activities of novel bronsted acidic ionic liquid catalysts. Catal. Lett., 139, 17-25.

18.Tajbakhsh, M., Mohammadpoor-Baltork, I., Alimohammadi, K. (2003). An Efficient and Selective Solid State Oxidation of Thiols to Disulfides with Quinolinium Fluorochromate on Silica Gel. Monat. Chem., 134, 1571-1575.

19. Sarrafi, Y., Mohadeszadeh, M., Alimohammadi, K. (2009). Microwave- assisted chemoselective copper-catalyzed amination of o-chloro and o-bromobenzoic acids using aromatic amines under solvent free conditions. Chin. Chem. Lett., 20, 784-788.

20. Azizi, S. N., ghasemi, Sh., Chiani, E. (2013). Nickel/mesoporous silica (SBA-15) modified electrode: An effective porous material for electrooxidation of methanol. Electrochim. Acta, 88, 463-472.

21. Su, W., Li, J., Zheng, Z., Shen, Y. (2005). One-pot synthesis of dihydropyrimidiones catalyzed by strontium (II) triflate under solvent-free conditions. Tetrahedron Lett., 46, 60376040 .

22. Sun, Q., Wang, Y., Ge, Z., et al. (2004). A highly efficient solvent-free synthesis of dihydropyrimidinones catalyzed by zinc chloride. Synthesis, 7, 1047-1051.

23. Zorkun, I. S., Sarac, S., Semra, C., et al. (2006). Synthesis of 4-aryl-3, 4-dihydropyrimidin$2(1 H)$-thiones derivatives as potential calcium channel blockers. Bioorg. Med. Chem., 14, 85828589.

24. Litvic, M., Vecenaj, I., Ladisic, Z. M. et al. (2010). First application of hexaaquaaluminium (III) tetrafluoroborate as a mild, recyclable, non-hygroscopic acid catalyst in organic synthesis: a simple and efficient protocol for the multigame scale synthesis of 3, 4-dihydropyrimidinones by Biginelli reaction. Tetrahedron, 66, 3463-3471.

25. Shaabani, A., Bazgir, A., Teimouri, F. (2003). Ammonium chloride- catalyzed one- pot synthesis of 3, 4- dihydropyrimidin-2(1H) - ones under solvent-free conditions. Tetrahedron Lett., $44,857-859$.

26. Salehi, P., Dabiri, M., Zolfigol, M. A., et al. (2003). Silica sulfuric acid: an efficient and reusable catalyst for the one-pot synthesis of 3, 4-dihydropyrimidin-2(1H) -ones. Tetrahedron Lett., 44, 2889-2891.

27. Lu, J., Bai, Y., Wang, Z., et al. (2000). One- pot synthesis of 3, 4-dihydropyrimidin-2(1H)ones using lanthanum chloride as a catalyst. Tetrahedron Lett., 41, 9075-9078. 
28.Ranu, B. C., Hajra, A., Jana, U. (2000). Indium (III) Chloride-Catalyzed One-Pot Synthesis of Dihydropyrimidinones by a Three-Component Coupling of 1,3-Dicarbonyl Compounds, Aldehydes, and Urea: An Improved Procedure for the Biginelli Reaction. J. Org. Chem., 65, 62706276.

29. Memarian, H. R., Sabzyan, H., Farhadi, A. (2009). Microwave-assisted oxidation of 3, 4dihydropyrimidin-2(1H) -ones. Zeitschrift fur Naturforschung, 64, 532-540.

30. Fu, N-Y., Yuan, Y- F., Cao, Z., et al. (2002). Indium (III) bromide-catalyzed preparation of dihydropyrimidiones: improved protocol conditions for the niginelli reaction. Tetrahedron, 58, 4801-4807. 